\title{
Compact Dual-Band Dual-Polarized Antenna for MIMO LTE Applications
}

\author{
Lila Mouffok, Anne Claire Lepage, Julien Sarrazin, and Xavier Begaud \\ Department Comelec Institut Mines Telecom, Telecom ParisTech, LTCI CNRS UMR 5141, 46 Rue Barrault, \\ 75634 Paris Cedex 13, France \\ Correspondence should be addressed to Lila Mouffok, lila.mouffok@telecom-paristech.fr
}

Received 15 May 2012; Revised 18 July 2012; Accepted 6 September 2012

Academic Editor: Minh-Chau Huynh

Copyright (c) 2012 Lila Mouffok et al. This is an open access article distributed under the Creative Commons Attribution License, which permits unrestricted use, distribution, and reproduction in any medium, provided the original work is properly cited.

A system of two dual-band dual-polarized antennas is proposed. It operates in two bands, 700 to $862 \mathrm{MHz}$ and 2.5 to $2.69 \mathrm{GHz}$, thereby making it suitable for LTE applications. The design is composed of two compact orthogonal monopoles printed close to each other to perform diversity in mobile terminals such as tablets or laptops. For each band, two orthogonal polarizations are available and an isolation higher than $15 \mathrm{~dB}$ is achieved between the two monopoles spaced by $\lambda_{0} / 10$ (where $\lambda_{0}$ the central wavelength in free space of the lower band). A good agreement is observed between simulated and experimental results. The antenna diversity capability is highlighted with the calculation of envelope correlation and mean effective gain for several antennas' positions in different environment scenarios.

\section{Introduction}

Deployment of existing and emerging wireless communication systems require a high-data-rate transmission, in order to satisfy the needs of multimedia applications on terminals. Multiple Input Multiple Output (MIMO) applications have been suggested as an effective way to increase the channel capacity by exploiting multipath scattering effects.

MIMO technology is present in many recent wireless standards, such as Long Term Evolution (LTE), and will be implemented in mobile devices [1]. Several research works have proven the efficiency of two-antenna diversity on mobile terminals $[2,3]$. However, when the available space is limited, the use of a dual-polarized antenna is more suitable than two separated antennas [4]. A variety of dual-polarized antennas have been reported recently in which good dual-polarized radiation over a wide bandwidth [5] and high isolation between the feeding ports [6] have been achieved. However, these antennas are mainly designed for single-band operation [7] or for frequencies above $800 \mathrm{MHz}$ [8]. Most of the dual-band dual-polarized antennas proposed in literature exploit harmonics frequencies [9] or use techniques to generate additional resonances such as insertion slot [10]. But generally, it leads to a ratio between frequency bands below or equal to 2 and implies a dependence between the two frequency bands. Today, very few designs are reported for dual-band dual-polarized operations for the following bands: $700-862 \mathrm{MHz}$ and $2.5-$ $2.69 \mathrm{GHz}$. In this paper, we firstly present the design of a dual-band antenna which can provide a dual-polarization for each band, for LTE devices such as a tablet or a laptop. Then, we introduce an enhanced design in which the lower bandwidth has been increased and the mutual coupling between ports has been reduced in the two bands. The lower band is extended towards TV White Space (TVWS) band to provide radio-cognitive capabilities to the terminal [11].

Finally, the diversity performances of the proposed dualband dual-polarized antennas are evaluated through the envelope correlation $\left(\rho_{e}\right)$ and the mean effective gain in isotropic, indoor, and outdoor environments.

\section{Antenna Design}

As shown in Figure 1, the proposed structure is composed of two orthogonal monopoles with dimensions of 


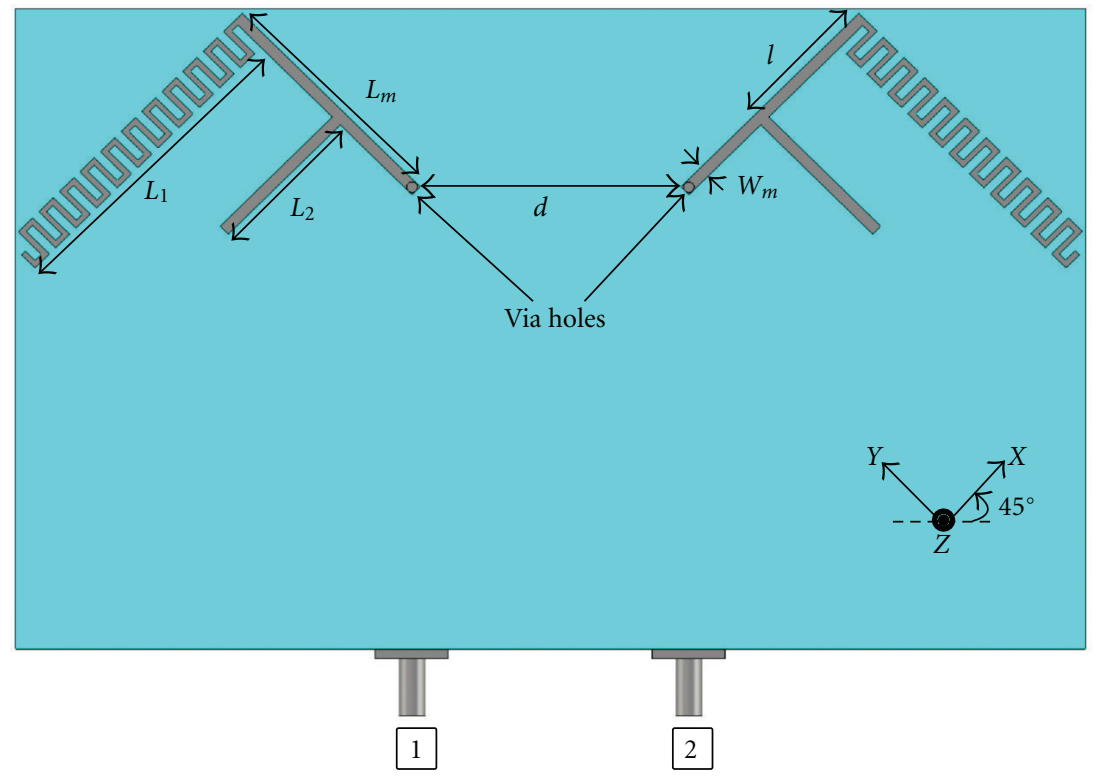

(a)

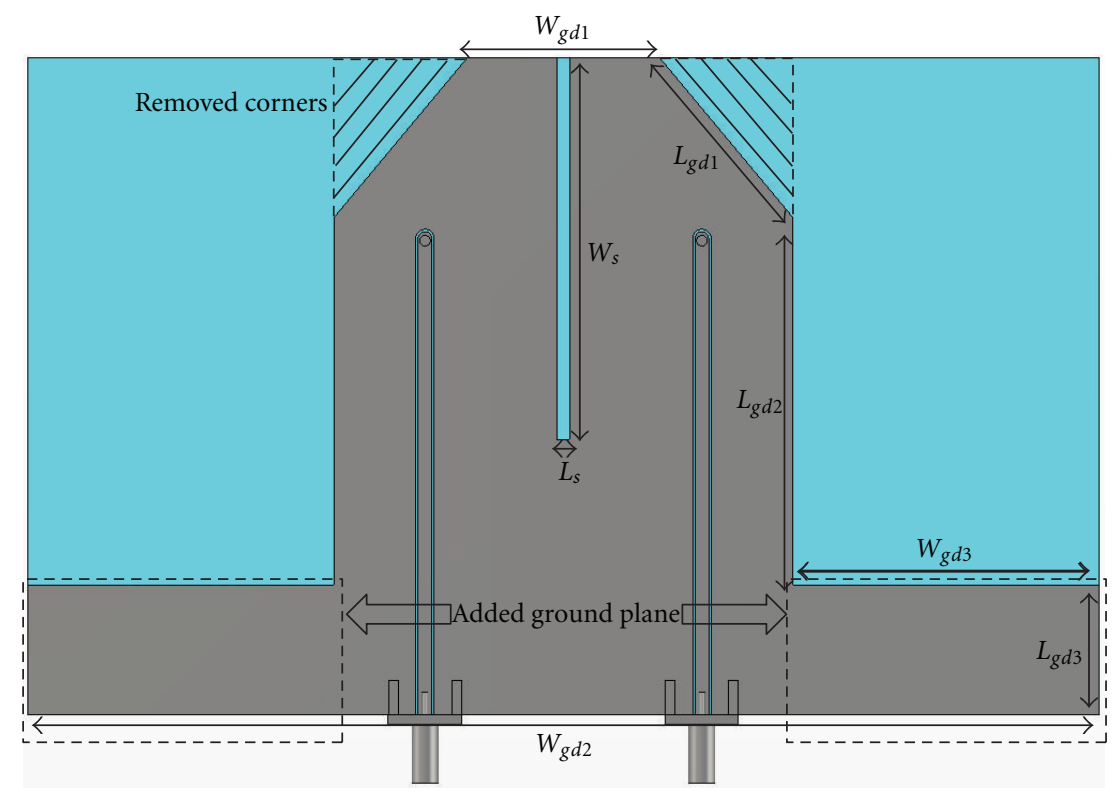

(b)

FIGURE 1: (a) Front view. (b) Back view of one meander bend ending antennas, with added ground plane and slot.

$L_{m} \times W_{m}=27.5 \times 1.5 \mathrm{~mm}^{2}$. The two monopoles are identical and chosen for their omni-directional radiations pattern, enabling them to receive signals whatever their orientation. They are printed on a $140 \times 83 \mathrm{~mm}^{2}$ low cost substrate (FR4: $\varepsilon_{r}=3.8 \pm 0.1, \tan \delta=0.02$, thickness of $0.7 \mathrm{~mm}$ ). Each monopole is connected to two bend endings: one bend ending is a meander line whose length is $L_{1}=43.3 \mathrm{~mm}$ operating at 790-862 MHz and the small one whose length is $L_{2}=23.5 \mathrm{~mm}$ operates at $2.5-2.69 \mathrm{GHz}$. The distance between the two bend endings is $l=14 \mathrm{~mm}$. This design allows to obtain independent frequency bands. The two monopoles are spaced by $d=36 \mathrm{~mm}$ which corresponds to $\lambda_{01} / 10$ for the lower band and $\lambda_{02} / 3$ for the higher band, where $\lambda_{01}$ is the free-space wavelength of the lower band central frequency $\left(f_{01}=826 \mathrm{MHz}\right)$ and $\lambda_{02}$ the free-space wavelength of the higher band central frequency $\left(f_{02}=\right.$ 2.59 GHz).

The monopoles are fed by two $50 \mathrm{ohms}$ coplanar waveguides (CPW), directly etched in the ground plane, as shown in Figure 1(b), in order to distance the connectors and to avoid perturbations on the measured radiation patterns. Each CPW has a line width of $1.8 \mathrm{~mm}$ and a gap of $0.33 \mathrm{~mm}$ 


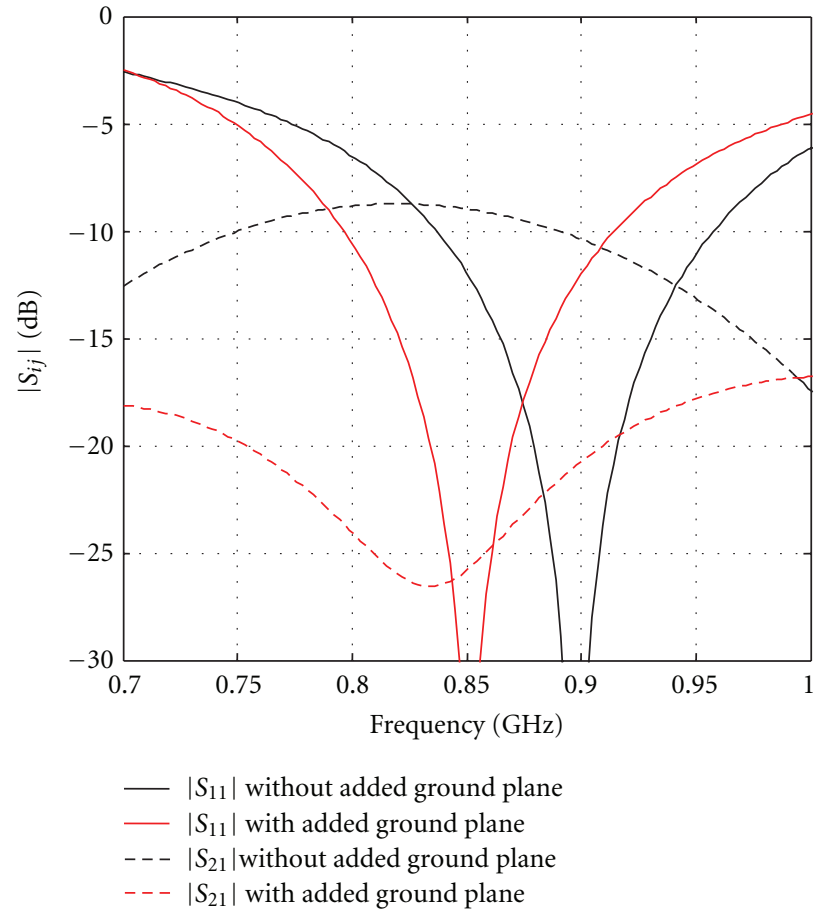

(a)

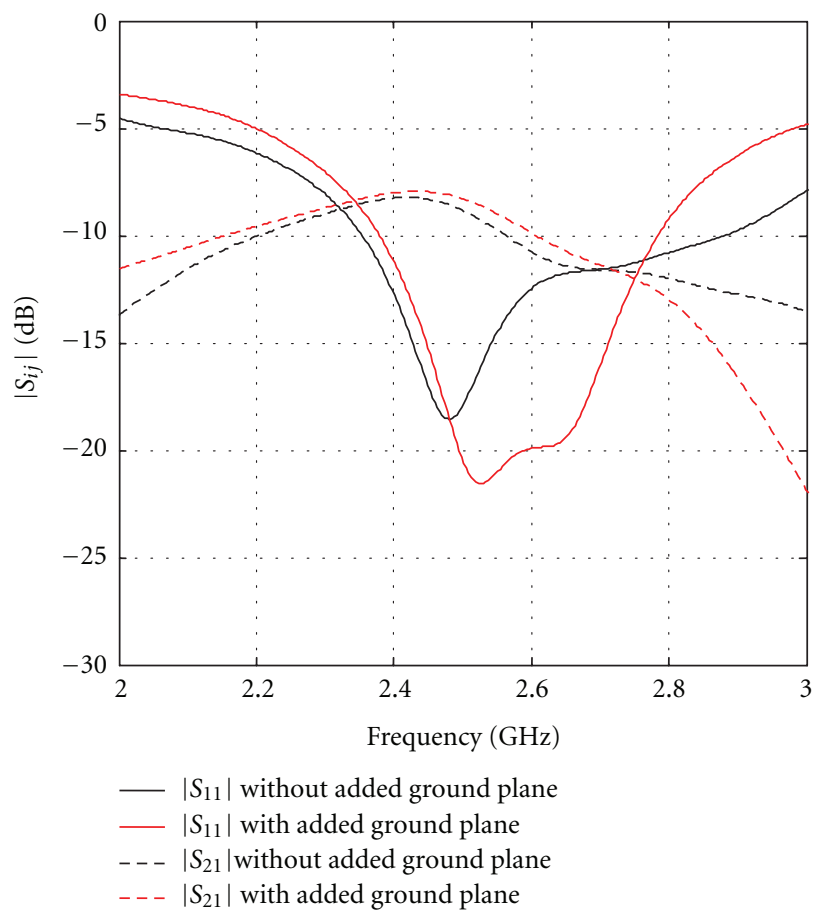

(b)

FIGURE 2: Simulated $\left|S_{i j}\right|$ parameters of one meander bend ending antennas without slot, with and without added ground plane: (a) lower band, (b) higher band.

with the ground plane. Monopoles are connected to CPW thanks to metallic via holes located as the extremity of each monopole as shown in Figure 1(a).

2.1. Ground Plane Geometry. Since the small bend ending is close to the ground plane extremities, it is sensitive to the path taken by currents along the ground plane. Therefore, a study of the upper part of the ground plane geometry is relevant. It is found that removing corners (shaded part in Figure 1(b)) provides an improvement of higher band matching, leading to optimized dimensions $W_{g d 1}=25 \mathrm{~mm}$, $L_{g d 1}=25 \mathrm{~mm}$, and $L_{g d 2}=46 \mathrm{~mm}$.

Coupling between the two antennas occurs via currents flowing from one antenna to the other one through the ground plane. It can be reduced by altering the ground plane to modify currents' path. Thus, the ground plane is extended without increasing the overall structure size, by adding on the lower part of the substrate, two rectangular shapes on either side with dimensions of each one $W_{g d 3}=40 \mathrm{~mm}$ and $L_{g d 3}=$ $17 \mathrm{~mm}$ (framed part in Figure 1(b)). Simulations have been performed with Transient Solver of CST Microwave Studio. Figure 2 shows a comparison between $\left|S_{i j}\right|$ parameters for designs without slot, with and without added ground plane in each band. Because of the structure's symmetry, only $\left|S_{11}\right|$ and $\left|S_{21}\right|$ are plotted. The matching bandwidth criterion is taken for a return loss less than $-10 \mathrm{~dB}$. With added ground plane, a shift of the lower band towards lower frequencies (from 0.9 to $0.85 \mathrm{GHz}$ ) is observed in Figure 2(a) without increasing the structure size. The bandwidths of the structure without added ground plane are: $837-957 \mathrm{MHz}(13.4 \%)$, 2.35-2.86 GHz (19.6\%), and for the structure with added ground plane are: $796-914 \mathrm{MHz}(13.8 \%), 2.38-2.78 \mathrm{GHz}$ $(15.5 \%)$. Regarding the isolation, it is largely reduced thanks to the added ground plane: $\left|\mathrm{S}_{21}\right|$ becomes below $-20 \mathrm{~dB}$ in the lower band. Indeed, a resonance has been introduced at the frequency where coupling occurs. However, the coupling remains high $\left(\left|S_{21}\right|<-7 \mathrm{~dB}\right)$ in the higher band as shown in Figure 2(b).

To improve isolation between ports in the higher band, a slot is etched in the ground plane while keeping the same distance between ports (d), as shown in Figure 1(a). The introduction of the slot produces an open circuit which stops the circulation of current from one radiating element to the other one [12]. The optimized structure has a length $L_{s}=34 \mathrm{~mm}$ and a width $W_{s}=1.4 \mathrm{~mm}$. Figure 3 shows a comparison of simulated $\left|S_{i j}\right|$ parameters of one meander bend ending antennas with added ground plane, with and without slot in the higher band. The introduction of the slot achieves an isolation improvement of $10 \mathrm{~dB}$ in the higher band, while it has no effect in the lower band. The bandwidth is slightly reduced but still covers the desired band. Thus, optimization of the two degrees of freedom which are the slot dimensions and rectangular shapes ground plane dimensions leads to a high isolation in the two frequency bands.

2.2. Radiating Element. In order to increase the bandwidth of the lower band towards the TVWS band, two bend endings 


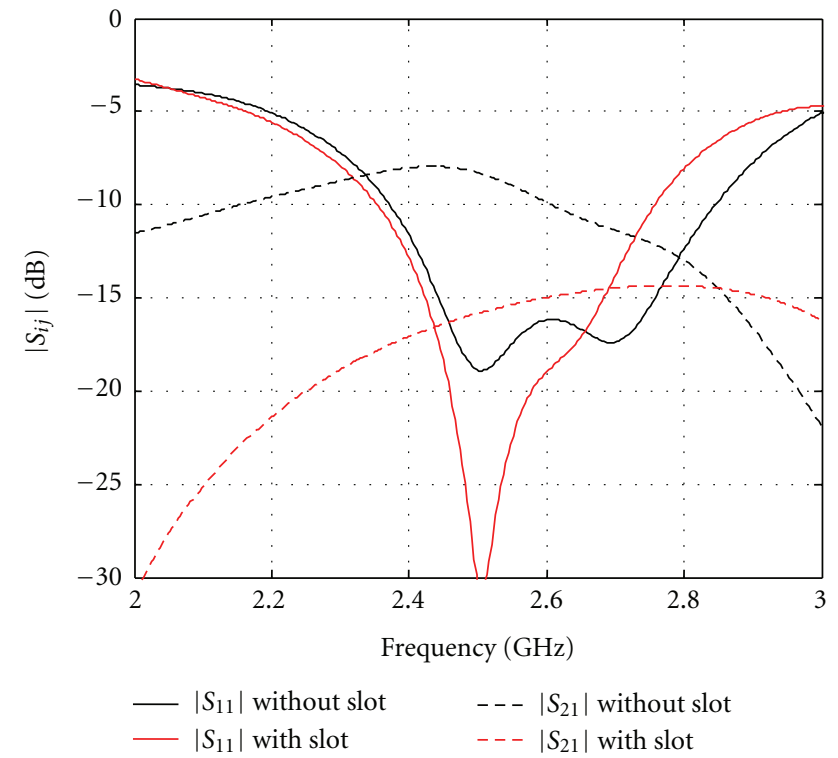

Figure 3: Simulated $\left|S_{i j}\right|$ parameters of one meander bend ending antennas with added ground plane, with and without slot in the higher band.

are added below the initial meander line to provide additional resonances close to each other. These two meanders are out of sync to provide a single wide band. Moreover, the three lines are connected to each other to extend the bandwidth towards lower frequencies. After optimization with Transient Solver of CST Microwave Studio, the distance between each meander is $s=7 \mathrm{~mm}$ as shown in Figure 5 and the overall size of three bend endings antennas with added ground plane and slot becomes $150 \times 90 \mathrm{~mm}^{2}$.

Figure 4 shows the comparison between S-parameters of one and three bend endings antennas with added ground plane and slot. Matching bandwidth criterion is taken for $\left|S_{11}\right|<-10 \mathrm{~dB}$. It is seen that the bandwidth is enhanced towards lower frequencies. Indeed, the relative bandwidth for the structure with one bend ending is $9.8 \%(786-867 \mathrm{MHz})$ and $21.9 \%(692-862 \mathrm{MHz})$ for the structure with 3 bend endings. While keeping almost the same electrical length of the structure, the relative bandwidth has been improved by $12 \%$. Indeed, the overall size is $0.35 \lambda_{\text {low }} \times 0.21 \lambda_{\text {low }}$ for three meander bend ending antennas $\left(\lambda_{\text {low }}\right.$ : the free space wavelength at $692 \mathrm{MHz})$ when it is $0.37 \lambda_{\text {low }}^{\prime} \times 0.22 \lambda_{\text {low }}^{\prime}$ for one meander bend ending antennas $\left(\lambda_{\text {low }}^{\prime}\right.$ : the free space wavelength at $786 \mathrm{MHz}$ ).

\section{Prototype and Measurement}

A prototype of three bend endings antennas with added ground plane and slot described previously has been realized. Monopoles and the ground plane with CPW are located on opposite sides of the same substrate and can be seen simultaneously on Figure 5 because of the transparency of the FR4 substrate. Simulated and measured S-parameters are

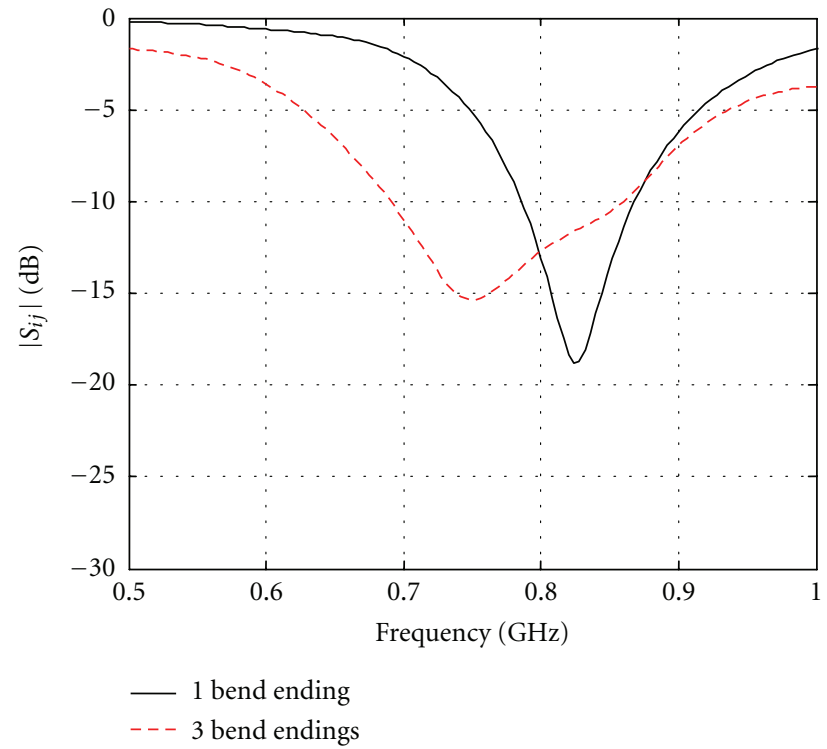

Figure 4: Simulated $\left|S_{11}\right|$ parameters of one and three meander bend endings antennas with added ground plane and slot in the lower band.

compared in Figure 6. Simulations results are in good agreement with measurement. The measurement results show that the antenna operates in two bands $\left(\left|S_{11}\right|<-10 \mathrm{~dB}\right)$ : the lower band extends from $700-880 \mathrm{MHz}(21.9 \%)$ and the higher one from $2.51-2.72 \mathrm{GHz}(8 \%)$. In these two bands, the two monopoles are satisfactorily uncoupled with an isolation $\left|S_{21}\right|$ below $-15 \mathrm{~dB}$ within the higher band and from 770 to $880 \mathrm{MHz}$. At the beginning of the lower band, the isolation remains acceptable and is below $-10 \mathrm{~dB}$. The simulated total efficiency of the structure, which takes into account all losses, has been evaluated: it varies from 83 to $97 \%$ in the lower band and from 74 to $87 \%$ in the higher band as shown in Figures 7 and 8 .

Figure 9 compares the simulated and measured copolar and cross-polar radiation patterns in the $E$ plane ( $Y Z$ plane) and $H$ plane ( $X Z$ plane), respectively. Because both ports are symmetrical, we only represent radiation patterns for port number 1 while port 2 is loaded by $50 \mathrm{ohms}$. For both planes and both bands, it is found that the simulated and the measured co-polar radiation patterns are in good agreement. The maximum simulated realized gain is $2.5 \mathrm{~dB}$ at $778 \mathrm{MHz}$ and $5 \mathrm{~dB}$ at $2.6 \mathrm{GHz}$. The measured cross-polar level is about $10 \mathrm{~dB}$ lower than the copolar level in the lower band but in the higher one, the polarization purity is deteriorated. It is probably due to the proximity of the meander bend endings to the small one.

To further investigate the diversity, the simulated radiation patterns of each radiating element in the $X Y$ plane for the two bands are plotted in Figure 10 (one port is excited while the other one is loaded by $50 \mathrm{ohms}$ ). Thanks to a good agreement observed in Figure 9 between simulations and measurement, only simulations results are presented. As it can be observed for the lower band, the directions 


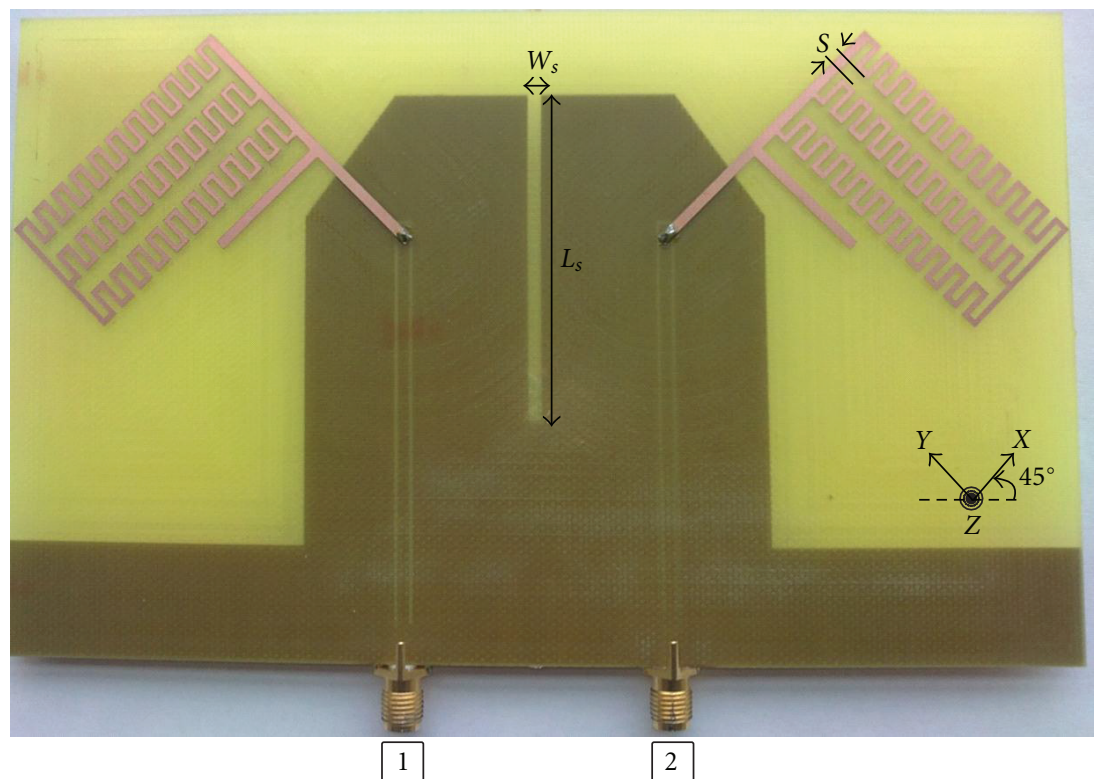

FIgURE 5: A photograph of the prototype with the three meander bend endings antennas.

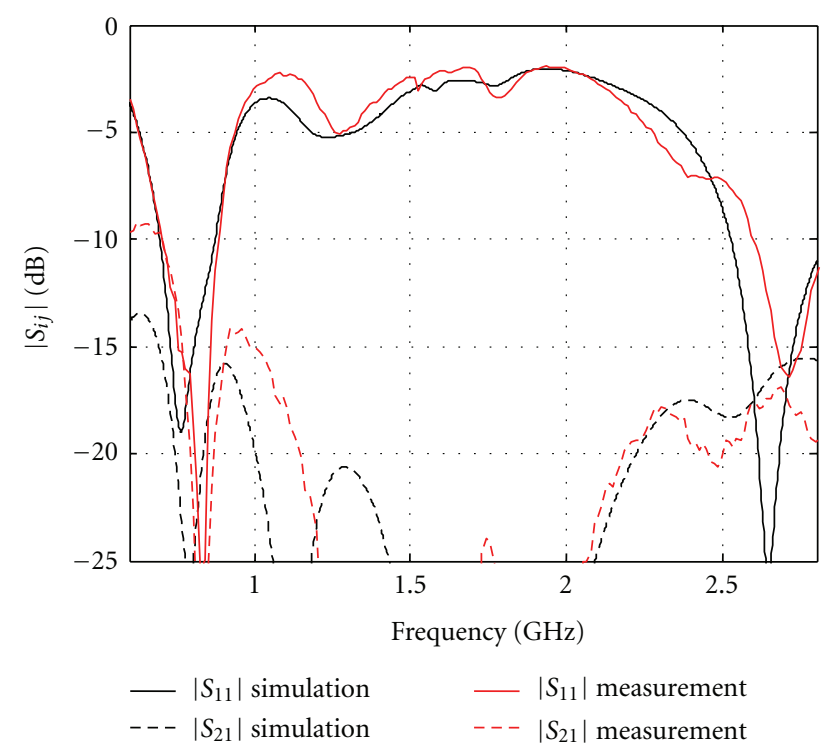

Figure 6: Simulated and measured $\left|S_{i j}\right|$ parameters of the optimized three bend endings antennas with added ground plane and slot.

of the pattern maxima are close to orthogonal, leading to good pattern diversity. Each antenna presents monopolelike radiation patterns. Indeed surface currents are weak on the bend endings. For the higher band, even if patterns are not orthogonal, one monopole presents minimum gain directions where the other one has a maximum gain, except for the directions $\theta= \pm 45^{\circ}$. This is well-suited to provide high diversity capabilities.

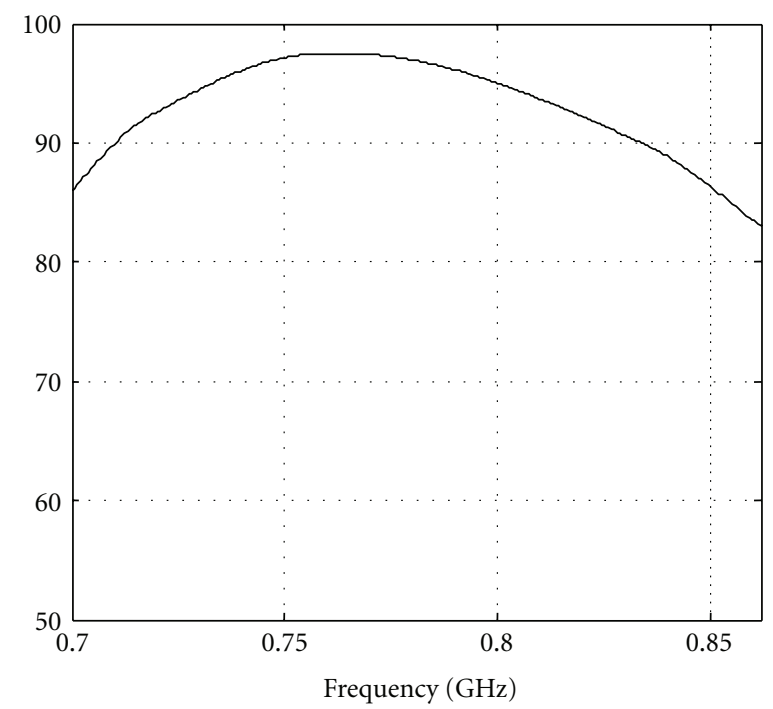

Figure 7: Simulated total efficiency in the lower band for the optimized three bend endings antennas with added ground plane and slot.

\section{Evaluation of the Diversity Performance}

The diversity performance of a mobile's antenna system can be affected by the environment in which the device is located [13]. Therefore, in this section, we evaluate the diversity performance of the proposed three bend endings antennas with added ground plane and slot, by calculating the envelope correlation coefficient $\left(\rho_{e}\right)$ and the mean effective gain (MEG) taking into account the propagation environment. 


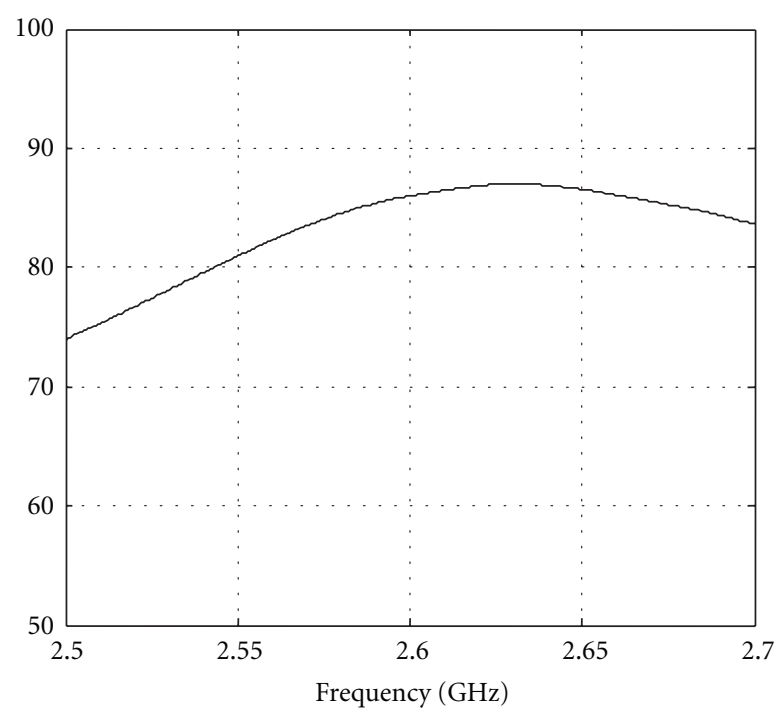

FIGURE 8: Simulated total efficiency in the higher band for the optimized three bend endings antennas with added ground plane, and slot.

The envelope correlation $\rho_{e}$ quantifies the similarity between the radiation patterns of the two monopoles. The lower the correlation, the better the diversity performance.
Vaughan and Andersen show in [13] that the coefficient can be expressed by

$$
\rho_{e}=\frac{\left|\int_{\Omega}\left(\operatorname{XPD}_{1 \theta} E_{2 \theta}^{*} p_{\theta}+E_{1 \varphi} E_{2 \varphi}^{*} p_{\varphi}\right) d \Omega\right|^{2}}{\int_{\Omega}\left(\operatorname{XPD} E_{1 \theta} E_{1 \theta}^{*} p_{\theta}+E_{1 \varphi} E_{1 \varphi}^{*} p_{\varphi}\right) d \Omega \int_{\Omega}\left(\operatorname{XPD}_{2 \theta} E_{2 \theta}^{*} p_{\theta}+E_{2 \varphi} E_{2 \varphi}^{*} p_{\varphi}\right) d \Omega} .
$$

$E_{1 \theta}(\Omega), E_{1 \varphi}(\Omega), E_{2 \theta}(\Omega), E_{2 \theta}(\Omega)$ are simulated complex electric fields along $\theta$ and $\varphi$ radiated by the antenna fed by two different ports. The solid angle $\Omega$ is defined by $\theta[0: \pi]$ in elevation and $\varphi[0: 2 \pi]$ in azimuth. $p_{\theta}(\Omega)$ and $p_{\varphi}(\Omega)$ are the Angle-of-Arrival (AoA) distributions of incoming waves. The parameter XPD is the cross-polarization discrimination of the incident field and is defined as XPD $=S_{\theta} / S_{\varphi}$ (where $S_{\theta}$ and $S_{\varphi}$ represent the average power along the spherical coordinates $\theta$ and $\varphi$ ).

The environment depends strongly on the angles of arrival distribution and on XPD. The most common distributions proven by measurements are Gaussian $(G)$ and Laplacian (L) distributions [14]. Thus, we consider different distributions in elevation, while in azimuth plane (XY plane) the distribution is uniform, as demonstrated by two measurement campaigns in the literature $[14,15]$.

To obtain more realistic results, different environments are considered. Each environment is characterized by typical values of XPD, mean angle of incident wave distribution $\left(\theta_{i}\right)$, and standard deviation of wave distribution $(\sigma)$ [16]. These values were deduced from several measurements [14-16] for different environments: isotropic, indoor, and outdoor. The isotropic environment is defined by XPD $=0 \mathrm{~dB}, p_{\theta}(\Omega)=$ $p_{\varphi}(\Omega)=1$, the indoor (In) environment by XPD $=1 \mathrm{~dB}$, $\theta_{i}=20^{\circ}, \sigma=30^{\circ}$, and the outdoor (Out) environment by $\mathrm{XPD}=5 \mathrm{~dB}, \theta_{i}=10^{\circ}, \sigma=15^{\circ}$.

As antennas will be implemented on a mobile terminal, a study of the effect of the antennas orientation on the correlation has been done. Three configurations of rotations have been studied: rotation of antenna around axis $A$, and around axis $B$ for two initial positions: horizontal and vertical, as shown in Figure 11.

For each configuration, the envelope correlation coefficient for the three meander bend endings antennas with added ground plane and slot has been calculated from simulated radiation patterns. Minimum and maximum values at center frequencies of the two bands $777 \mathrm{MHz}$ and 2.6 $\mathrm{GHz}$ are reported in Table 1 .

For isotropic environment, a very low correlation is observed, in the two bands as a result of good matching $\left(\left|S_{11}\right|<-10 \mathrm{~dB}\right)$, a high isolation level $\left(\left|S_{21}\right|<-10 \mathrm{~dB}\right)$, and orthogonality between radiation patterns especially in the lower band. In addition, polarization diversity is naturally achieved because of the orthogonal positions of both antennas.

For the other cases, maximum values of the correlation envelope coefficient $\rho_{e}$ are close to 0.5 for outdoor environment, whatever the distribution. Indeed, the incoming waves are mainly along $E_{\theta}$ which implies less diversity in some antenna's position.

When XPD gets close to $0 \mathrm{~dB}$ (indoor environment: $\mathrm{XPD}=1 \mathrm{~dB}), E_{\theta}$ and $E_{\varphi}$ values are almost the same. Because these two components are uncorrelated by definition and because each antenna receives preferentially one of each component, the correlation is getting low. 


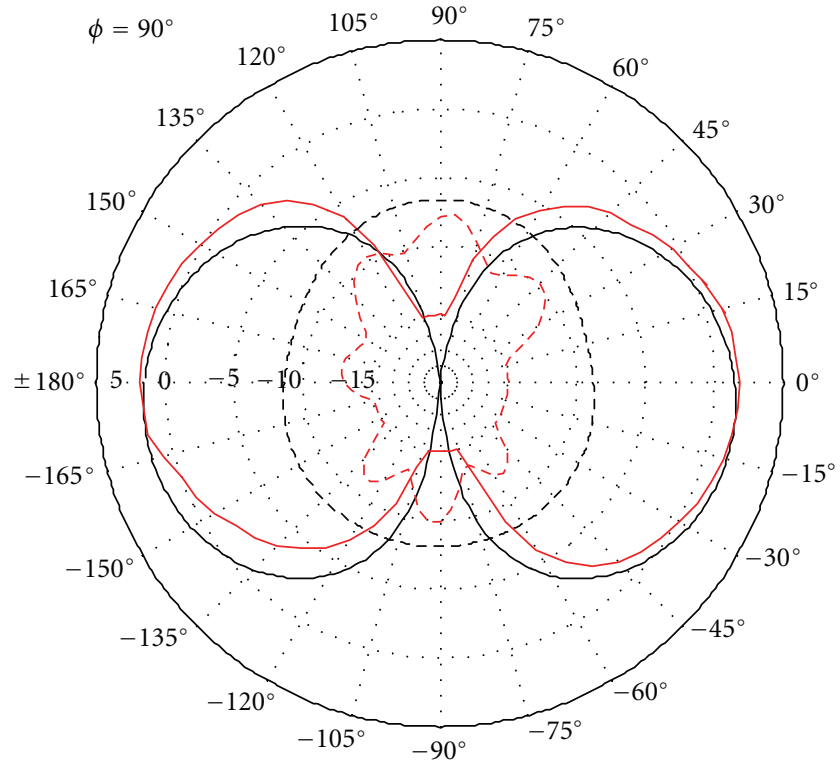

(a)

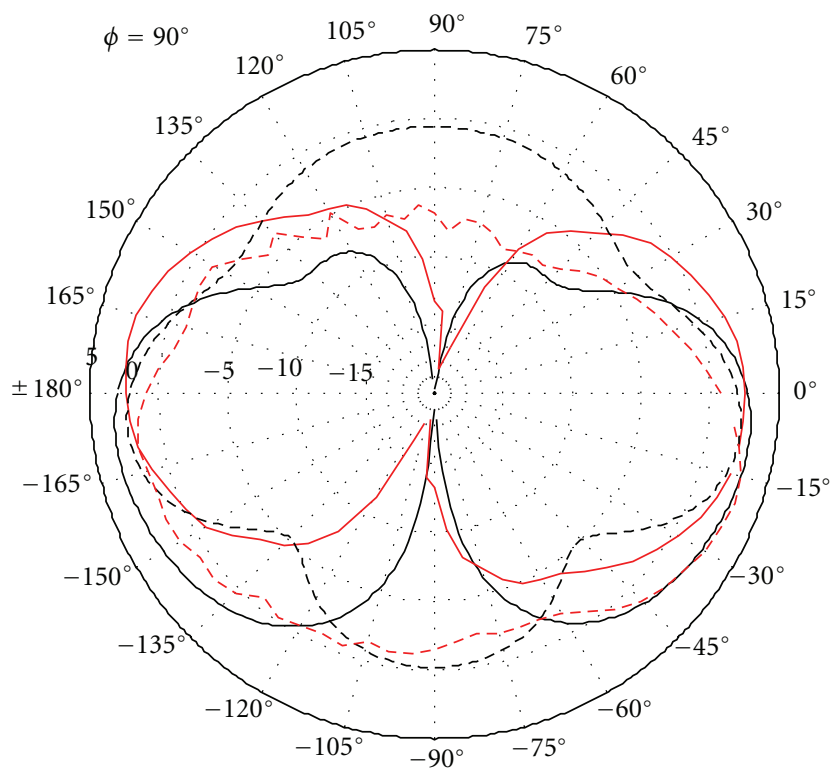

(c)

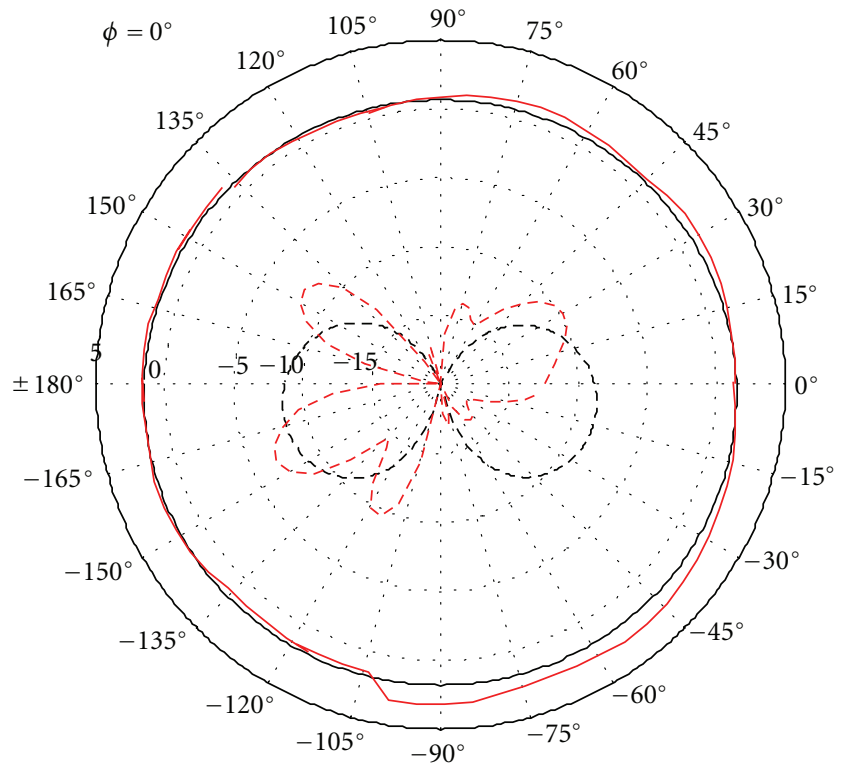

(b)

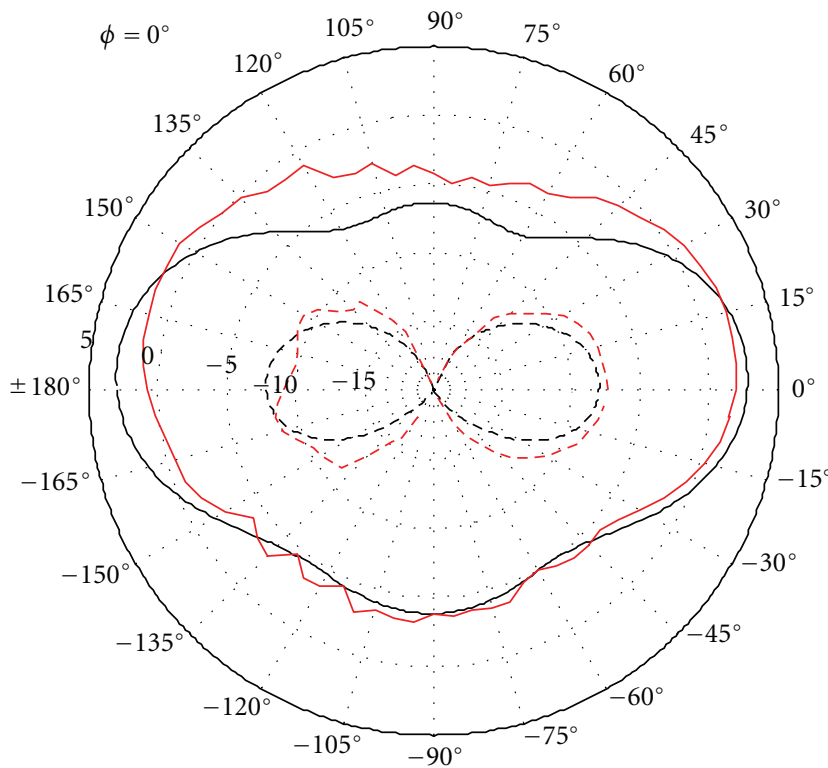

- Copolar simulation
- Copolar measurement
-- Cross-polar simulation
--- Cross-polar measurement

(d)

FIGURE 9: Simulated and measured radiation patterns for port $1(\mathrm{~dB})$ : (a) in the $E$ plane ( $Y Z$ plane) at $778 \mathrm{MHz}$, (b) in the $H$ plane ( $X Z$ plane) at the $778 \mathrm{MHz}$, (c) in the $E$ plane ( $Y Z$ plane) at $2.6 \mathrm{GHz}$, and (d) in the $H$ plane ( $X Z$ plane) at $2.6 \mathrm{GHz}$.

For rotation around axis $A$, minimum values of $\rho_{e}$ are obtained for position at which one antenna receives only $E_{\theta}$ component of the incoming waves while the other one only $E_{\varphi}$ component.

For rotation around axis $B$, for both configurations (b and $c$ ), minimum values are obtained when the two radiating elements are positioned on $A B$ plane. Indeed, at these positions, the radiation diversity is exploited as shown in Figure 10, and thus a low correlation is obtained.

Finally, for most configurations, envelope correlation coefficient is less than 0.5 which provides high diversity capabilities [13]. This result has been achieved thanks to the two orthogonal and identical antennas which are spatially separated. It can provide for either or both spatial 


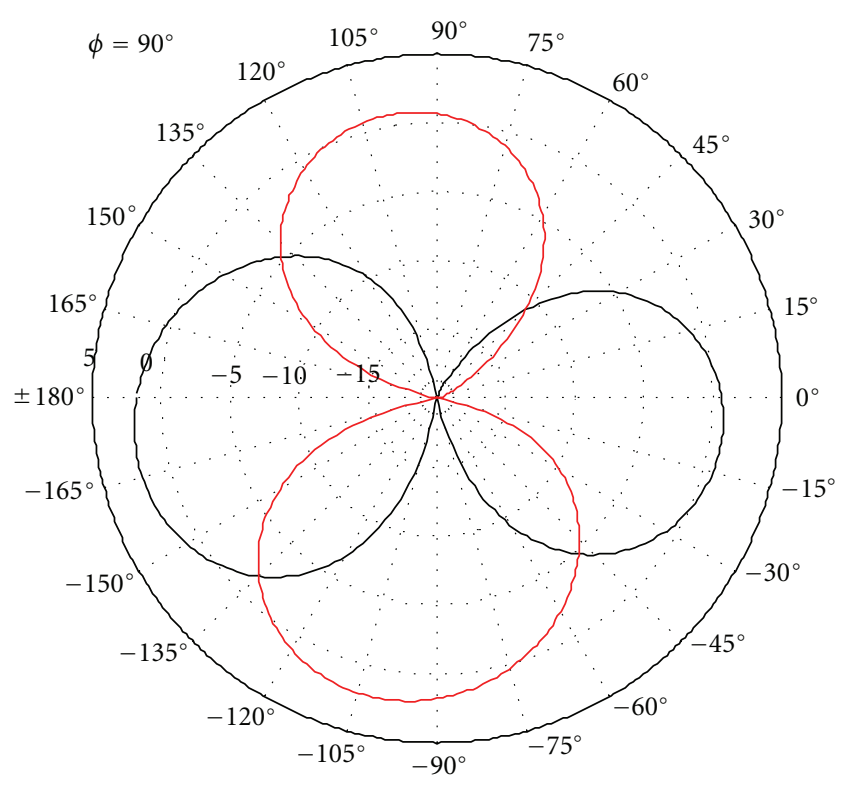

(a)

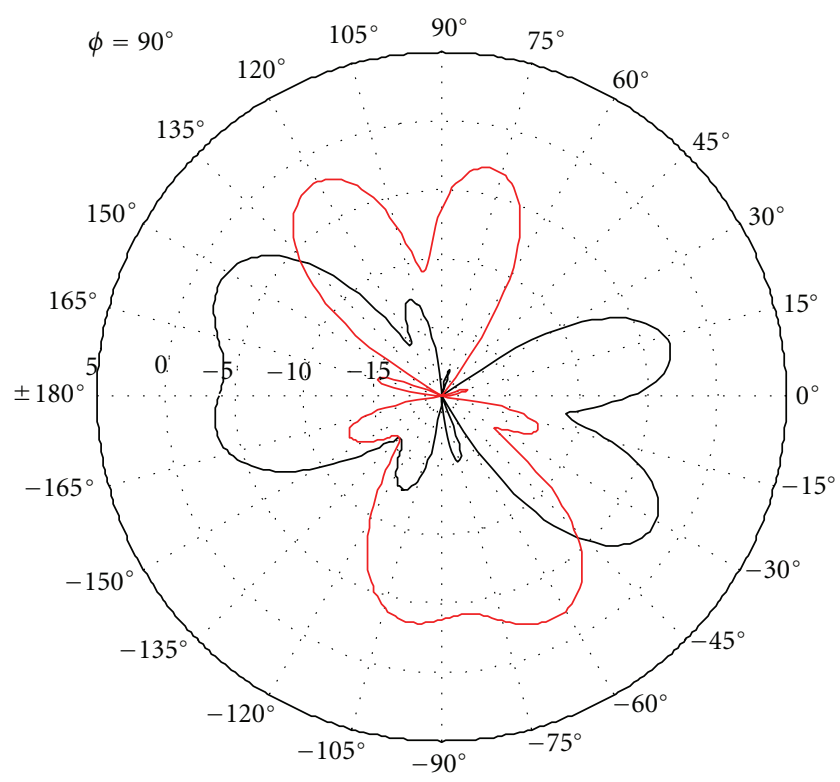

- Port 1

- Port 2

(b)

Figure 10: Simulated realized gain $(\mathrm{dB})$ on the $X Y$ plane for the two bands: (a) at $778 \mathrm{MHz}$ (b) at $2.6 \mathrm{GHz}$.

and pattern diversity. In addition, polarization diversity is available in the $Z$-direction.

In the following part, we evaluate the MEG which was introduced by Taga [17]. It is defined as the ratio between the mean received power of antennas over the random route and the total mean incident power. When each monopole receives the same quantity of power, the MEG ratio $(R)$ of the two antennas is equal to one, which means that no performance deterioration is expected due to some power imbalance [18].

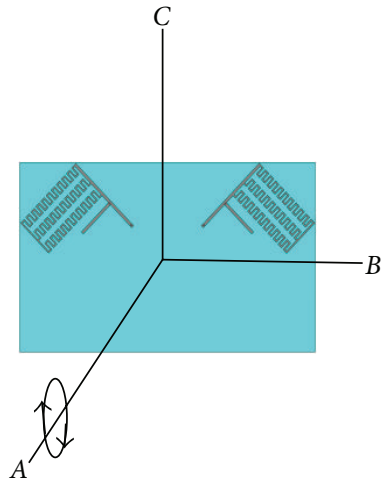

(a)

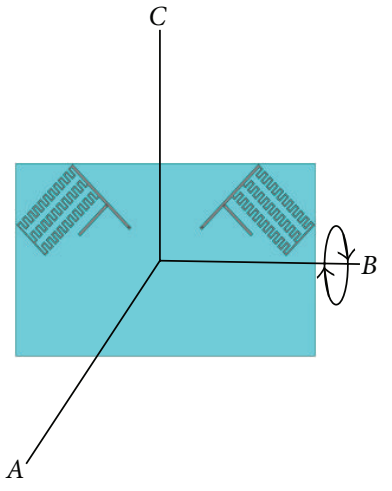

(b)

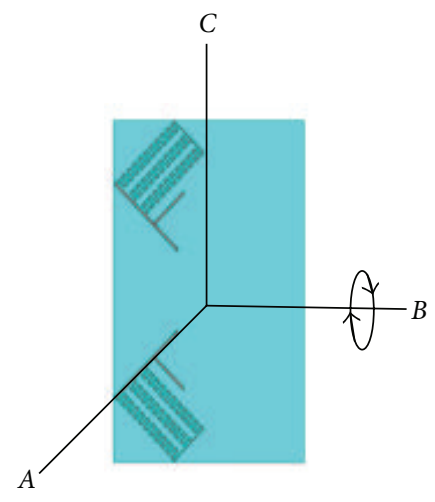

(c)

FIGURE 11: Rotation of antenna: (a) around axis $A$, (b) around axis $B$ (horizontal antenna position), and (c) around axis $B$ (vertical antenna position).

The mathematical expression is given by the following equation:

$$
\mathrm{MEG}=\int_{\Omega}\left(\frac{\mathrm{XPD}}{\mathrm{XPD}+1} G_{\theta} P_{\theta}+\frac{1}{\mathrm{XPD}+1} G_{\varphi} P_{\varphi}\right) d \Omega,
$$

where $G_{\theta}$ and $G_{\varphi}$ are the $\theta$ and $\varphi$ components of the antenna power gain pattern, respectively. The calculated mean effective gains of the monopoles from simulated radiation patterns at $777 \mathrm{MHz}$ and $2.6 \mathrm{GHz}$ are presented in Table 2.

The Maximum values of the ratio $(R)$ of $\mathrm{MEG}_{1}$, determined at port 1 , over $\mathrm{MEG}_{2}$, determined at port 2, are equal to 1 , which satisfy an equal contribution of the two monopoles to receive the same quantity of power. The proposed structure is completely symmetric, and the Gaussian and Laplacian angular distributions are taken only along the elevation as presented in [15]. In addition, the incident power in the outdoor environment (or indoor) is concentrated around $10^{\circ}$ (or $20^{\circ}$ ) above the horizon with an aperture of $30^{\circ}\left(\right.$ or $\left.60^{\circ}\right)$, and for these directions both antennas receive an equal amount of power.

Minimum values of ratio $(R)$ are obtained for positions at which the $E_{\theta}$ (or $E_{\varphi}$ ) components of the two antennas have different levels in the directions of incident power. 
TABLE 1: Coefficients of correlation for the two bands for all environments of the proposed structure.

\begin{tabular}{|c|c|c|c|c|c|}
\hline \multirow{2}{*}{ Rotation } & \multirow{2}{*}{ Distribution } & \multicolumn{2}{|c|}{$777 \mathrm{MHz}$} & \multicolumn{2}{|c|}{$2.6 \mathrm{GHz}$} \\
\hline & & $\rho_{e_{\min }}$ & $\rho_{e_{\max }}$ & $\rho_{e_{\min }}$ & $\rho_{e_{\max }}$ \\
\hline Whatever & Isotropic & \multicolumn{2}{|c|}{$7.10^{-5}$} & \multicolumn{2}{|c|}{$4.10^{-3}$} \\
\hline \multirow{4}{*}{ around $A$} & G-In & 0.02 & 0.10 & $10^{-3}$ & 0.08 \\
\hline & G-Out & 0.20 & 0.42 & $7.10^{-4}$ & 0.39 \\
\hline & L-In & 0.07 & 0.16 & $10^{-5}$ & 0.22 \\
\hline & L-Out & 0.26 & 0.49 & $3.10^{-4}$ & 0.51 \\
\hline \multirow{4}{*}{ around $B$ (horizontal position) } & G-In & $7.10^{-4}$ & 0.10 & $5.10^{-4}$ & $8.10^{-3}$ \\
\hline & G-Out & $10^{-3}$ & 0.42 & $4.10^{-5}$ & $1.10^{-2}$ \\
\hline & L-In & $2.10^{-3}$ & 0.16 & $10^{-5}$ & $3.10^{-3}$ \\
\hline & L-Out & $3.10^{-3}$ & 0.46 & $10^{-4}$ & $5.10^{-3}$ \\
\hline \multirow{4}{*}{ around $B$ (vertical position) } & G-In & $5.10^{-5}$ & 0.05 & $2.10^{-4}$ & 0.07 \\
\hline & G-Out & $10^{-4}$ & 0.40 & $4.10^{-3}$ & 0.39 \\
\hline & L-In & $2.10^{-4}$ & 0.10 & $8.10^{-5}$ & 0.22 \\
\hline & L-Out & $6.10^{-4}$ & 0.49 & $7.10^{-4}$ & 0.54 \\
\hline
\end{tabular}

TABLE 2: MEG ratio $(R)$ for the two bands for all environments of the proposed structure.

\begin{tabular}{lccccc}
\hline \multirow{2}{*}{ Rotation } & Distribution & \multicolumn{2}{c}{$777 \mathrm{MHz}$} & \multicolumn{2}{c}{$2.6 \mathrm{GHz}$} \\
& & $R_{\min }$ & $R_{\max }$ & $R_{\min }$ & $R_{\max }$ \\
\hline Whatever & Isotropic & & 1 & & \multicolumn{2}{c}{1} \\
\hline \multirow{3}{*}{ around $A$} & G-In & 0.70 & 1 & 0.78 & 1 \\
& G-Out & 0.35 & 1 & 0.71 & 1 \\
& L-In & 0.63 & 1 & 0.46 & 1 \\
& L-Out & 0.30 & 1 & 0.58 & 1 \\
\hline \multirow{3}{*}{ around $B$} & G-In & 0.99 & 1 & 0.99 & 1 \\
(horizontal position) & G-Out & 0.97 & 1 & 0.98 & 1 \\
& L-In & 0.99 & 1 & 0.99 & 1 \\
& L-Out & 0.96 & 1 & 0.98 & 1 \\
\hline \multirow{2}{*}{ around $B$} & G-In & 0.94 & 1 & 0.80 & 1 \\
(vertical position) & G-Out & 0.84 & 1 & 0.63 & 1 \\
& L-In & 0.85 & 1 & 0.33 & 1 \\
& L-Out & 0.70 & 1 & 0.48 & 1 \\
\hline
\end{tabular}

For example, if antenna 1 presents a low $E_{\theta}$ component where antenna 2 a high one, an unbalanced power is obtained.

For most configurations, ratio $(R)$ is greater than 0.5 which is acceptable to provide high diversity capabilities [18].

\section{Conclusion}

In this paper, a compact dual-band, dual-polarized antenna for LTE applications is proposed, with an extension of the lower band towards TV White Space band, to provide radiocognitive capabilities to the terminal. A design provides dual polarizations in both of the bands: $700-862 \mathrm{MHz}$ and $2.5-$ $2.69 \mathrm{GHz}$ with good impedance matching $\left(\left|S_{11}\right|<-10 \mathrm{~dB}\right)$.

Measurement results are in good agreement with simulated ones. In addition, good performances are obtained by calculating the envelope correlation coefficient and the MEG ratio for several antennas' positions in different environments: isotropic, indoor, and outdoor. For most configurations, it is found that the system satisfies the condition $\rho_{e}<0.5$ and $\mathrm{MEG}_{1} / \mathrm{MEG}_{2}>0.5$. Thus, the presented design is suitable for MIMO communication applications, and thus enables the SNR value at the terminal side to be maximized.

\section{Acknowledgment}

The research leading to these results has received funding from the European Community's Seventh Framework Program (FP7/2007-2013) under Grant agreement SACRA no. 249060 .

\section{References}

[1] 3rd Generation Partnership Project, Technical Specification Group Radio Access Network, Evolved Universal Terrestrial Radio Access (E-UTRA) Radio Resource Control (RRC), Protocol Specification, 3GPP TS 36. 331.

[2] R. G. Vaughan, "Polarization diversity in mobile communications," IEEE Transactions on Vehicular Technology, vol. 39, no. 3, pp. 177-186, 1990.

[3] K. Ogawa and T. Uwano, "Diversity antenna for very small $800-\mathrm{MHz}$ band portable telephones," IEEE Transactions on Antennas and Propagation, vol. 42, no. 9, pp. 1342-1345, 1994.

[4] J. W. Wallace, M. A. Jensen, A. L. Swindlehurst, and B. D. Jeffs, "Experimental characterization of the MIMO wireless channel: data acquisition and analysis," IEEE Transactions on Wireless Communications, vol. 2, no. 2, pp. 335-343, 2003.

[5] S. Hienonen, A. Lehto, and A. V. Raisanen, "Simple broadband dual-polarized aperture-coupled microstrip antenna," in Proceedings of the IEEE Antennas and Propagation Society International Symposium, vol. 2, pp. 1228-1231, Orlando, Fla, USA, August 1999.

[6] P. Brachat and J. M. Baracco, "Printed radiating element with two highly decoupled input ports," Electronics Letters, vol. 31, no. 4, pp. 245-246, 1995.

[7] Y. L. Kuo and K. L. Wong, "Dual-polarized monopole antenna for WLAN application," in Proceedings of the IEEE Antennas 
and Propagation Society International Symposium, vol. 4, pp. 80-83, June 2002.

[8] C. Yang, Y. Yao, J. Yu, and X. Chen, "Novel compact multiband MIMO antenna for mobile terminal," International Journal of Antennas and Propagation, vol. 2012, Article ID 691681, 9 pages, 2012.

[9] T. W. Chiou and K. L. Wong, "A compact dual-band dualpolarized patch antenna for 900/1800-MHz cellular systems," IEEE Transactions on Antennas and Propagation, vol. 51, no. 8, pp. 1936-1940, 2003.

[10] K. S. Kim, T. Kim, and J. Choi, "Dual-frequency aperturecoupled square patch antenna with double notches," Microwave and Optical Technology Letters, vol. 24, no. 6, pp. 370-374, 2000.

[11] SACRA European Project, (FP7/ 2007-2013), http://www.ictsacra.eu/.

[12] K. J. Kim, W. G. Lim, and J. W. Yu, "High isolation internal dual-band planar inverted-F antenna diversity system with band-notched slots for MIMO terminals," in Proceedings of the 36th European Microwave Conference (EuMC'06), pp. 14141417, Manchester, UK, September 2006.

[13] R. G. Vaughan and J. B. Andersen, "Antenna diversity in mobile communication," IEEE Transactions on Vehicular Technology, vol. 36, no. 4, pp. 149-172, 1987.

[14] K. Kalliola, K. Sulonen, H. Laitinen, O. Kivekäs, J. Krogerus, and P. Vainikainen, "Angular power distribution and mean effective gain of mobile antenna in different propagation environments," IEEE Transactions on Vehicular Technology, vol. 51, no. 5, pp. 823-838, 2002.

[15] F. Adachi, M. T. Feeney, A. G. Williamson, and J. D. Parsons, "Crosscorrelation between the envelopes of $900 \mathrm{MHz}$ signals received at a mobile radio base station site," Proceedings of IEE on Communications, Radar and Signal Processing Part F, vol. 133, no. 6, pp. 506-512, 1986.

[16] Z. Ying, T. Bolin, V. Plicanic, A. Derneryd, and G. Kristensson, "Diversity antenna terminal evaluation," in Proceedings of the IEEE Antennas and Propagation Society International Symposium and USNC/URSI Meeting, pp. 375-378, July 2005.

[17] T. Taga, "Analysis for mean effective gain of mobile antennas in land mobile radio environments," IEEE Transactions on Vehicular Technology, vol. 39, no. 2, pp. 117-131, 1990.

[18] W. C. T. Brown, Antenna diversity for mobile terminal [Ph.D. thesis], University of Surrey, 2002, http://epubs.surrey .ac.uk/2125/. 

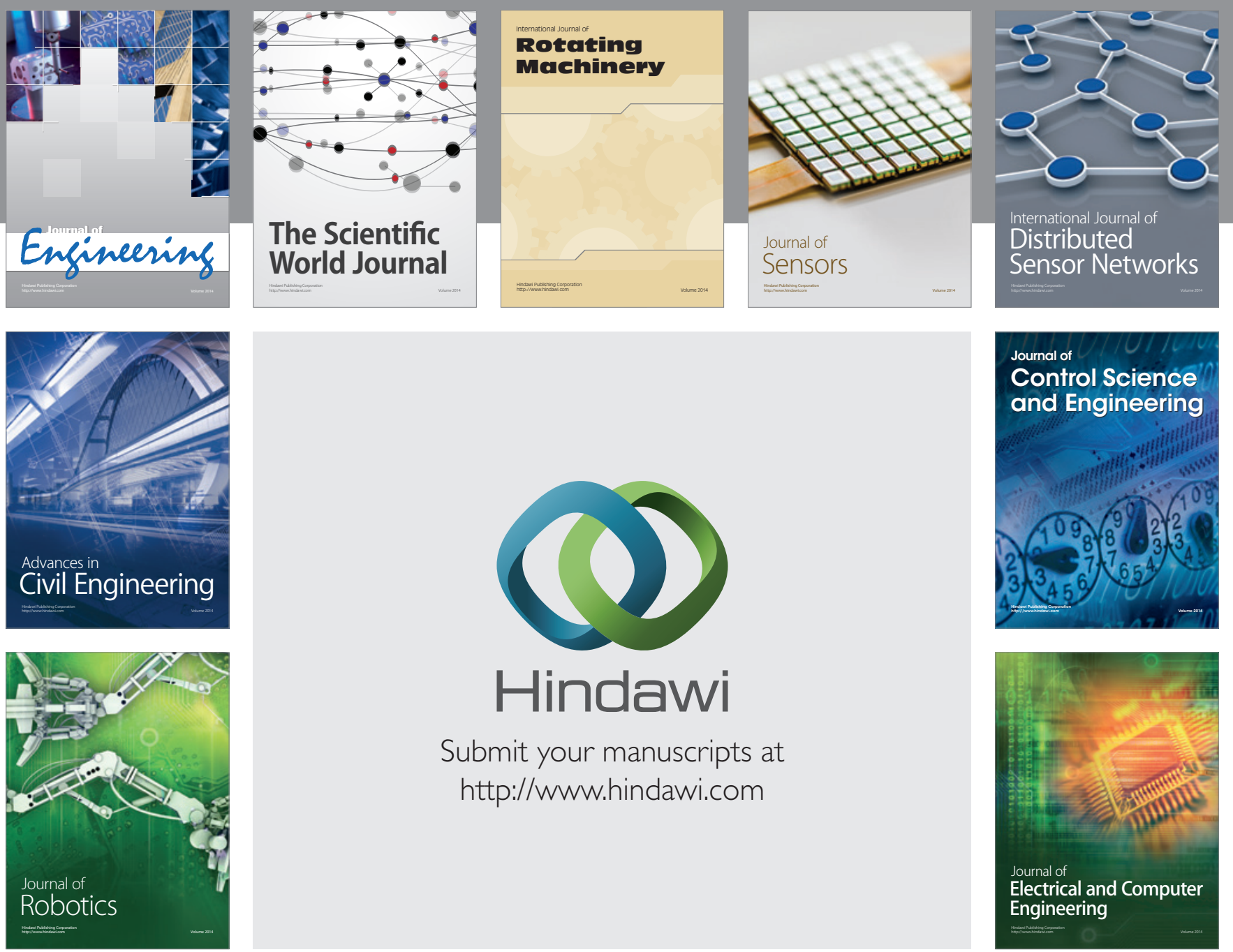

Submit your manuscripts at

http://www.hindawi.com
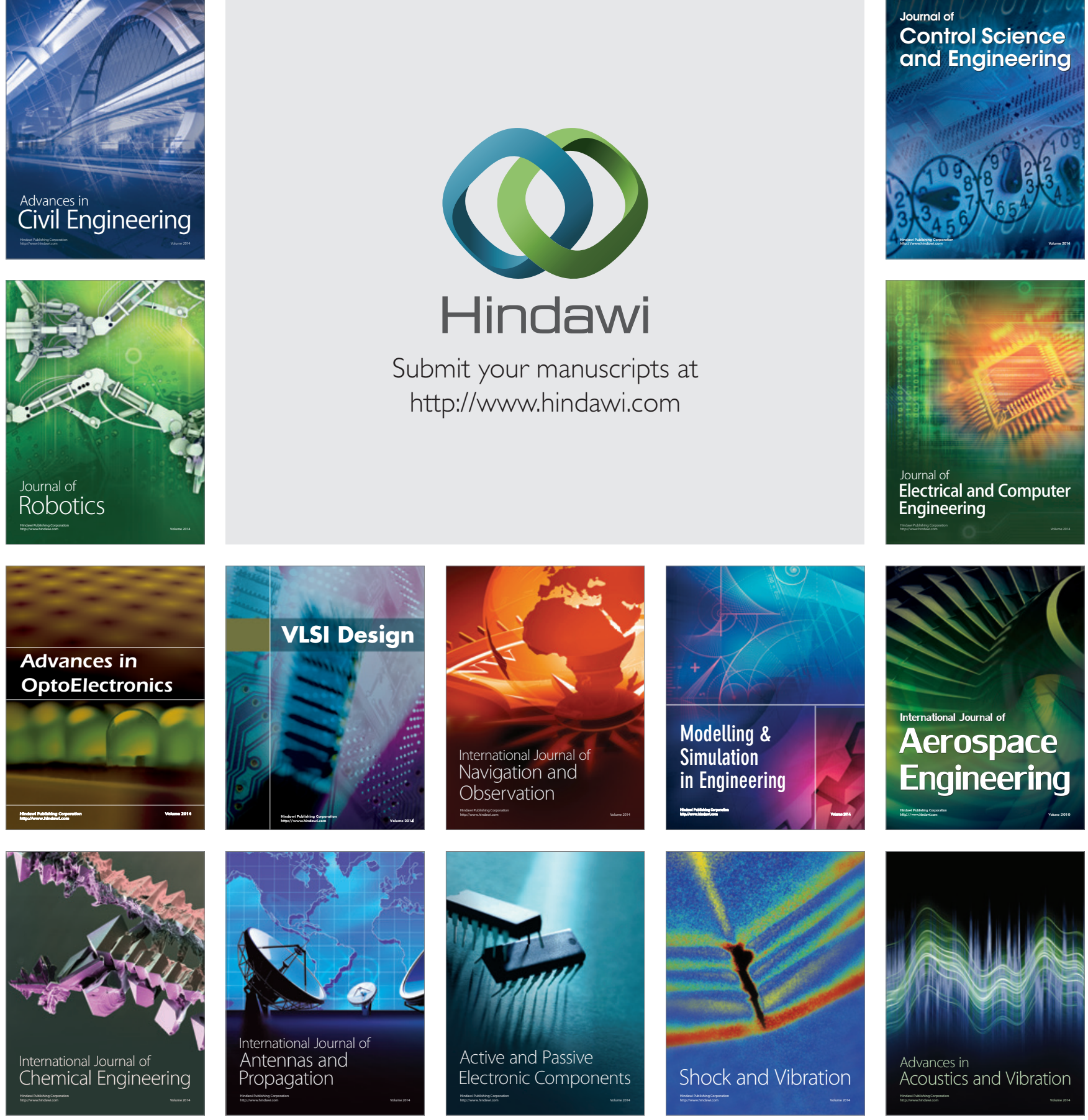